\title{
Flow visualization and simulation of the filling process during injection molding
}

\section{Guerrier, Patrick; Tosello, Guido; Hattel, Jesper Henri}

\section{Published in:}

C I R P - Journal of Manufacturing Science and Technology

Link to article, DOI:

10.1016/j.cirpj.2016.08.002

Publication date:

2017

Document Version

Peer reviewed version

Link back to DTU Orbit

\section{Citation (APA):}

Guerrier, P., Tosello, G., \& Hattel, J. H. (2017). Flow visualization and simulation of the filling process during injection molding. C I R P - Journal of Manufacturing Science and Technology, 16, 12-20.

https://doi.org/10.1016/j.cirpj.2016.08.002

\section{General rights}

Copyright and moral rights for the publications made accessible in the public portal are retained by the authors and/or other copyright owners and it is a condition of accessing publications that users recognise and abide by the legal requirements associated with these rights.

- Users may download and print one copy of any publication from the public portal for the purpose of private study or research.

- You may not further distribute the material or use it for any profit-making activity or commercial gain

- You may freely distribute the URL identifying the publication in the public portal 


\title{
FLOW VISUALIZATION AND SIMULATION OF THE FILLING PROCESS DURING INJECTION MOLDING
}

\author{
P. Guerriera, ${ }^{*}$, G. Toselloa, J. H. Hattel ${ }^{a}$ \\ a Department of Mechanical Engineering, Technical University of Denmark, \\ Produktionstorvet Building 425, 2800 Kgs. Lyngby \\ ${ }^{*}$ Corresponding author, email address: pagu@mek.dtu.dk
}

\begin{abstract}
To directly compare experimental moldings from an injection molding machine with simulations, a special mold has been produced with a glass window. The injection plane is perpendicular to the opening and closing planes, in order for the $55 \mathrm{~mm}$ thick glass window to be easily visible from the side. A high speed camera recording 500 frames per second was employed, and the mold had three thermocouples and two pressure sensors installed. The molded part is a $2 \mathrm{~mm}$ thick plate with a $0.5 \mathrm{~mm}$ thin section, which creates a characteristic V-shaped flow pattern. Two different materials were employed, namely ABS and a high viscosity PC. Simulations were performed using the actual machine data as input, including the injection screw acceleration. Furthermore, the nozzle and barrel geometries were included as a hot runner to capture the effect of compressibility of the material in front of the screw. These two had significant effects on the filling times and injection pressure calculated by the simulations. Other effects investigated included transient thermal management of the mold, pressure dependent viscosity and wall slip, but their effect were not remarkably large in this work. The obtained simulation results showed deviations within 10-30 ms (relative deviation in the order of 5-10\%) for the ABS and slightly more for the high viscosity PC in the range of $100-500 \mathrm{~ms}$ (relative deviation in the order of $20-30 \%$ ) on timings between different sections during filling.
\end{abstract}

Keywords: Glass Mold, High Speed Camera, Injection Molding, Simulations

\section{Introduction}

Direct flow visualization with the use of a high speed camera is a known methodology to analyze the flow behavior in injection molding and other processes. In several studies experiments have been conducted with different kinds of camera setups in the molding tools. Yang et al. [1] have employed flow visualization using a quartz glass window in a micro injection molding tool to investigate the flow of micro parts with high injection speed, using high speed camera able to capture videos with 1000 frames per second. The mold contained a reflection mirror to allow space for the placement of camera and thermocouples. The main purpose of the investigation was the micro injection molding process characterization. Other studies which also use a reflective mirror type mold construction are: Nian et al. [2] use previously mentioned mold however this time combining transparent and colored material to get further information of the flow. Han et al. [3] use a high speed camera capable of recording at 13,500 frames per second and a microscope, to investigate the filling of micro grooves on a glass prism insert. Hasegawa et al. [4] use a glass insert with 
branching runners in order to investigate the influences of inertia forces on melt filling behavior at high injection speed. Layser et al. [5] investigate hesitation and emphasizes the use of the packing phase due to the use of a window made of fused quartz glass, which was able to withstand a packing pressure of up to 50 MPa. Spares et al. [6] have modified a thermal imaging camera to increase the frames per seconds, to investigate the thermal field during injection of micro molding. Whiteside et al. [7] have also investigated micro molding with a high speed camera and sapphire glass insert in order to study different effects in micro injection molding. Yokoi et al. [8] use a high speed camera with a mold with a build in microscope in order to investigate micro cavities and the effect of injection speed. All the mentioned work so far, uses a reflective mirror and no simulation comparison.

Other researches in the field of flow visualization in injection molding have been performed using a different mold design that, instead of using a reflective mirror, included a transparent insert directly applied to the mold cavity which is oriented parallel to the injection direction, so the flow can be directly filmed from the side of the injection molding machine. Yanev et al. [9] [10] applied a sapphire insert to this type of mold design. The researchers have also performed a simulation comparison to verify the filling performance of the software. It was stated that the simulation is capable of giving good overall agreement with the obtained flow patterns, but significant difference was found during the beginning of the filling phase. Furthermore, Dvorak et al. [11] also used a direct observation of the flow with a high speed camera without making use of a reflective mirror, for the characterization of ceramic injection molding.

The main focus of this work will be on making an experimental setup similar to [9] [10] i.e. using a turned insert for direct visualization of the flow in the cavity, which is oriented parallel to the injection screw of the molding machine. However in the present work a more comprehensive study of the influencing parameters on the simulation results is carried out. Using pressure sensors and thermocouples in combination with a high speed camera, the goals are to capture and characterize the filling of the mold cavity, as well as to analyze and compare the flow pattern with simulation on a time basis. Deviations between experiments and simulation predictions will be studied. Different features in the software will be utilized to minimize the observed deviations in order to obtain the correct timings of the filling pattern. The use of a high speed camera with a glass mold will provide a direct comparison of the filling, with an increased accuracy with respect to more conventional validation techniques such as the short shots method.

\section{Experimental}

\subsection{Glass mold}

In order to compare simulations directly with the filling of the cavity, a special glass mold was manufactured. The injection plane is almost perpendicular to the opening and closing plane, in order for the glass window to be easily visible from the side. The reason for not being turned $90^{\circ}$, but instead $82^{\circ} \mathrm{C}$, was due to the opening and closing of the mold, to prevent a too close and tight fit and possible breakage of the glass, either in opening and closing of the mold or during the packing phase by applying the holding force. This mold design makes it particularly effortless to install any kind of recording device to the mold. The glass window is made of a $55 \mathrm{~mm}$ thick Borosilicate glass (width and height $60 \times 140 \mathrm{~mm}$ ), and it is capable of withstanding at least $130 \mathrm{MPa}$ in machine pressure during injection, and $50 \mathrm{MPa}$ during packing. It was 
tested to go up to $220 \mathrm{MPa}$ which did not break the glass, even though this pressure level was not used during the experiments. Due to the (almost) perpendicular injection plane, and a fairly large air vent thickness $(0.02 \mathrm{~mm})$, moldings could result in some flash during some of the experiments. The experimental setting including the two mold halves, the glass window and the placement of the high speed camera, as well as a schematic overview of the mold can be seen in Fig. 1.
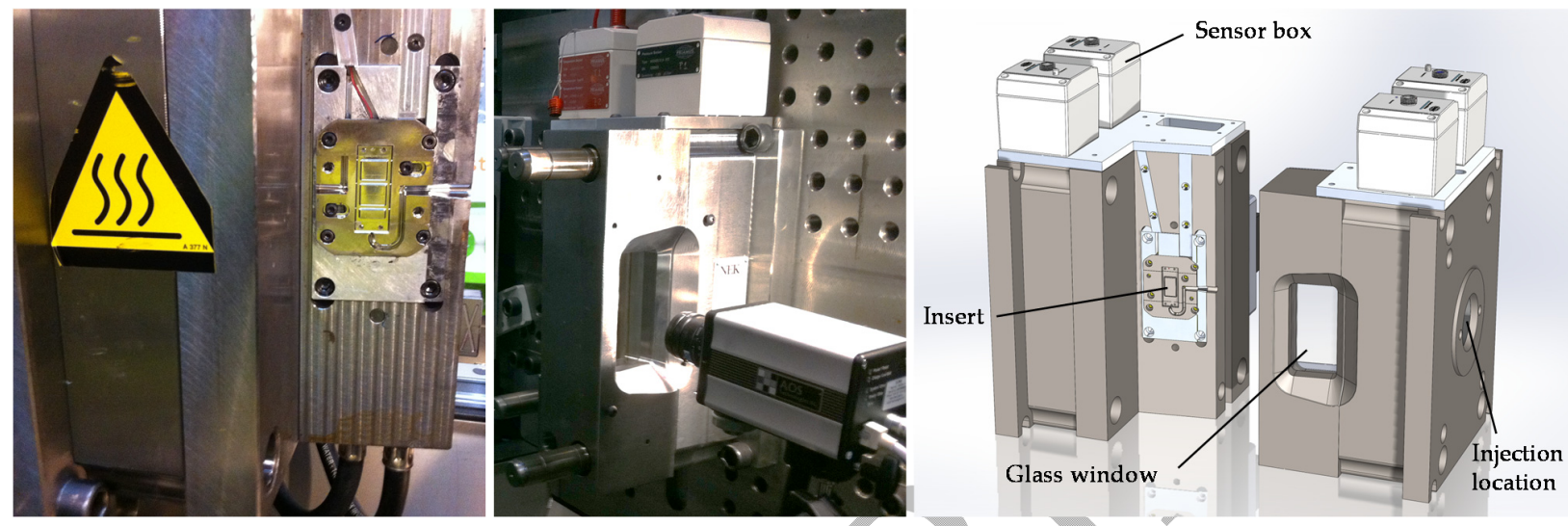

Fig. 1 - Two mold halves of the glass mold and schematic overview.

\subsection{Equipment and materials}

The employed high speed camera is an X-PRI produced by AOS Technologies AG (Fislisbach, Switzerland) and it is capable of recording $1280 \times 1024$ pixels images at 500 frames per second. The camera was easily installed inside the safety door of the injection molding machine, and attached to the track of the sliding door. Some additional lighting was also installed to improve the brightness of the recorded videos.

The mold is equipped with a total of five sensors. Three of the sensors are thermocouples, which are used to time when the melt is at the specific location. Furthermore, two pressure sensors are installed, one in the runner and one in the cavity just after the gate location. The two pressure sensors are used to determine when the polymer melt passes them, and to directly compare with the simulated pressure, in conjunction with the high speed recording system. Fig. 2 shows the location of the different sensors. In addition to the installed sensors, the machine data is also collected to be used as input for the simulation setup, in order to simulate the actual conditions in which the experiments are conducted.

The injection machine used is an Engel Victory 60 with a $35 \mathrm{~mm}$ diameter screw. Two different polymer materials were employed for the experiments, the first being a natural colored acrylonitrile butadiene styrene (ABS) grade with a melt flow index (MFI) of $7 \mathrm{~g} / 10 \mathrm{~min}$ and the second a gray colored high viscosity polycarbonate (HVPC) grade with a MFI of $34.3 \mathrm{~g} / 10 \mathrm{~min}$. Viscosity and pvT of both polymers were characterized in order to have accurate input for the simulations also in terms of material properties. 


\subsection{Test part}

The geometry used in the experiments can be seen in Fig. 2. It consists of an $18 \mathrm{~mm}$ wide and $45 \mathrm{~mm}$ long rectangle with a thickness of $2 \mathrm{~mm}$. It has a thin section in the middle of $0.5 \mathrm{~mm}$, which produces a flow which will slow down over the middle section, as the frozen layer starts to develop. This creates a characteristic V-shaped frozen front over the thin section, as the sides flow faster due to being thicker, hence not solidifying through the thickness and allowing melt to pass.

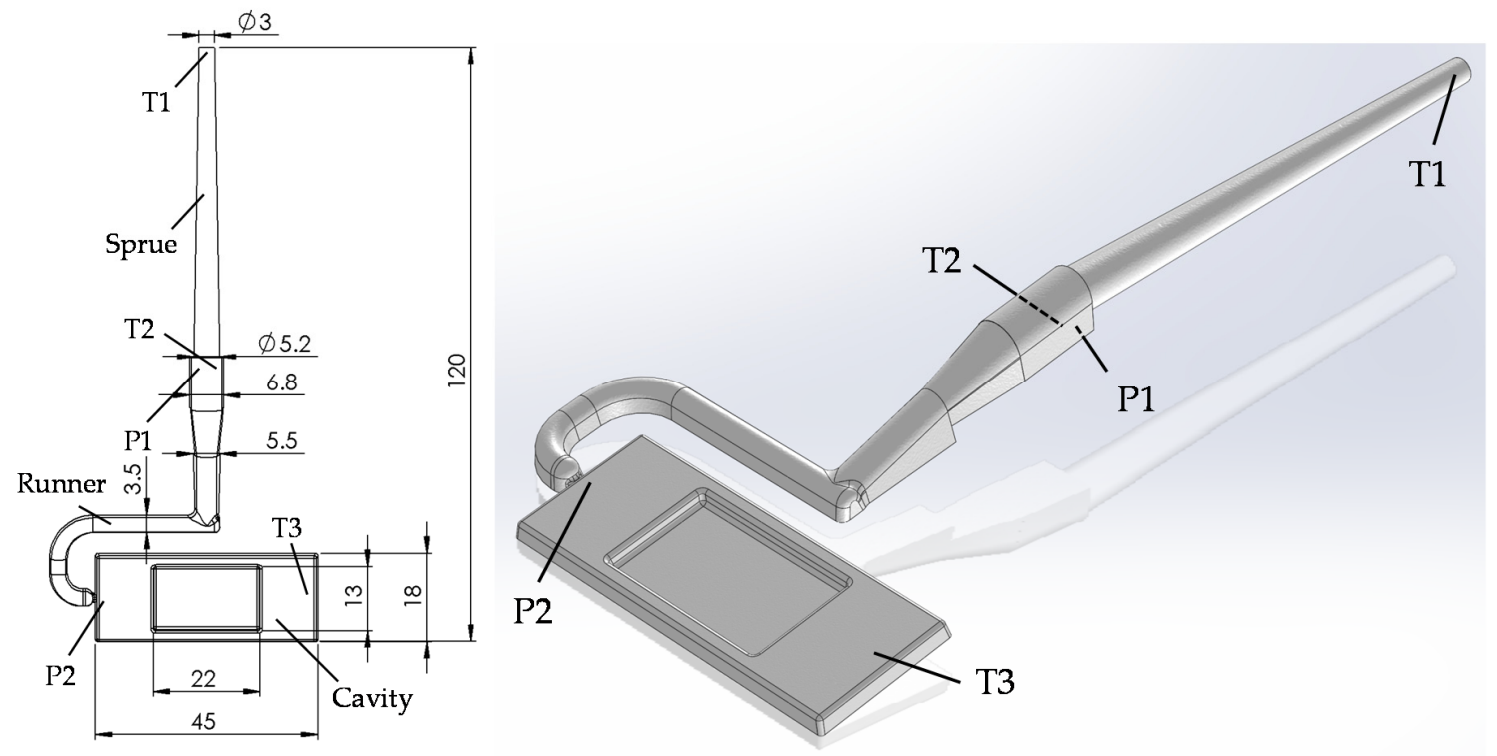

Fig. 2 - The test part with an overall thickness of $2 \mathrm{~mm}$, and a center section of $0.5 \mathrm{~mm}$, noted with sensor location. P1 and P2 indicate the pressure sensors; T1, T2 and T3 indicate the thermocouples.

\subsection{Setup}

The injection flow rate was specified in order for the filling (i.e. injection) time to be close to $1 \mathrm{~s}$. This target was achieved by adjusting the injection ram speed, which was close to constant at $10 \mathrm{~mm} / \mathrm{s}$ for ABS and 12 $\mathrm{mm} / \mathrm{s}$ for HVPC. The acceleration distance was $1.54 \mathrm{~mm}$ for ABS and $1.50 \mathrm{~mm}$ for HVPC, resulting in an acceleration time of approximately $0.15 \mathrm{~s}$ for both polymers. The filling was followed by $4 \mathrm{~s}$ of packing phase, with a holding pressure of $40 \mathrm{MPa}$ for the ABS and of $50 \mathrm{MPa}$ for the HVPC (stepped down to 35 $\mathrm{MPa}$ ). The cooling time was set at 15 seconds for both polymers. The melt temperature of the ABS was set to $237^{\circ} \mathrm{C}$ with cooling water at $17^{\circ} \mathrm{C}$, whereas the melt temperature of $\mathrm{HVPC}$ was set to $315^{\circ} \mathrm{C}$ and $95^{\circ} \mathrm{C}$ cooling water. The cooling channel is located in the opposite side of the mold half with the glass, together with the ejector system. The pressure sensors and thermocouple measurements from the experiments can be seen in Fig. 3. 

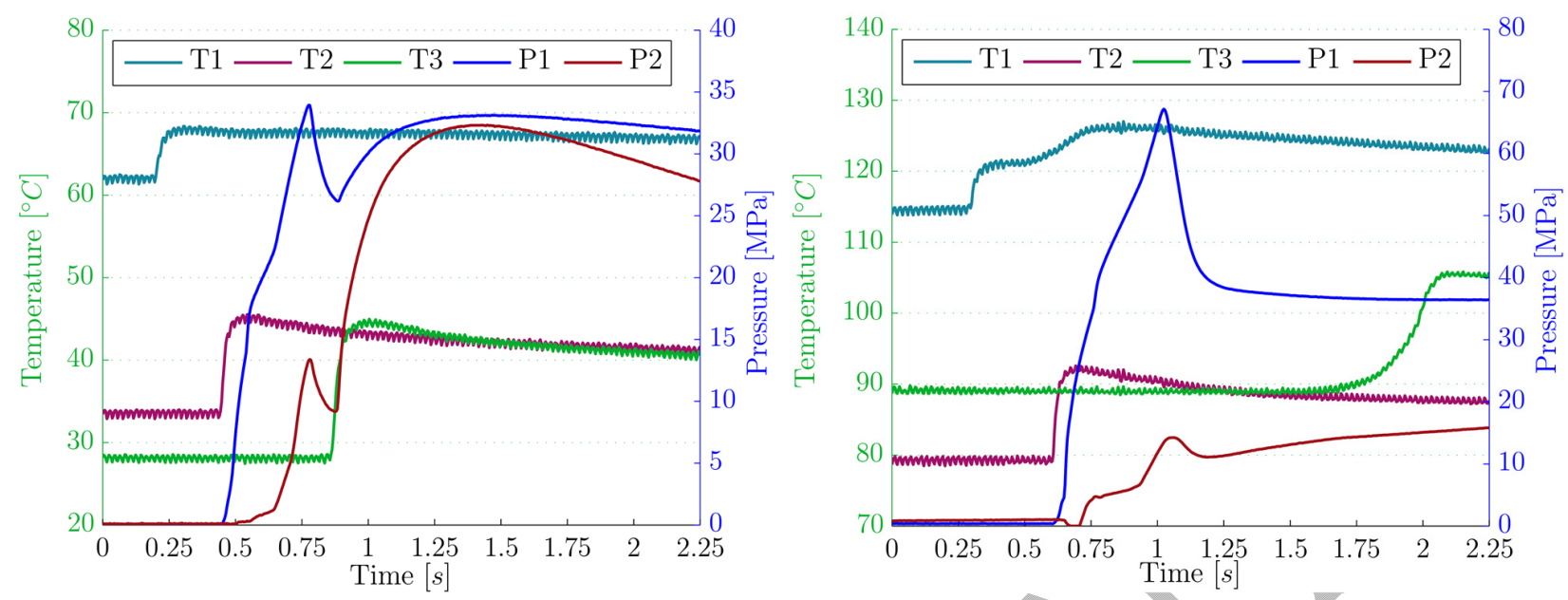

Fig. 3 - The three thermocouples and two pressure sensors signals during filling and part of the packing phase. Left: ABS. Right: HVPC.

\section{Simulations}

\subsection{Theoretical background}

For the flow simulations the principle of conservation of mass, momentum and energy is used for the governing equations:

$$
\begin{gathered}
\frac{\partial p}{\partial t}+\nabla \cdot \rho \boldsymbol{u}=0 \\
\frac{\partial}{\partial t}(\rho \boldsymbol{u})+\nabla \cdot(\rho \boldsymbol{u} \boldsymbol{u}-\boldsymbol{\sigma})=\rho \boldsymbol{g} \\
\boldsymbol{\sigma}=-p \boldsymbol{I}+\eta\left(\nabla \boldsymbol{u}+\nabla \boldsymbol{u}^{T}\right) \\
\rho c_{p}\left(\frac{\partial T}{\partial t}+\boldsymbol{u} \cdot \nabla T\right)=\nabla(k \nabla T)+\Phi \\
\Phi=\eta \dot{\gamma}_{e q}^{2}+\Delta \dot{H}
\end{gathered}
$$

where $\rho$ is the density, $\boldsymbol{u}$ is the velocity component, $p$ is the pressure, $\boldsymbol{\sigma}$ is the total stress tensor, $\dot{\gamma}$ is the shear rate, $\boldsymbol{g}$ is the body force, $c_{p}$ is the specific heat, $k$ is the thermal conductivity, $\Delta \dot{H}$ is the heat released under solidification and $\eta$ is the viscosity taken as the generalized WLF-Cross viscosity model:

$$
\eta(T, \dot{\gamma}, p)=\frac{\eta_{0}(T, p)}{1+\left(\eta_{0} \dot{\gamma} / \tau^{*}\right)^{1-n}}
$$

where $n$ is a power-law constant and $\tau^{*}$ is a transition stress. $\eta_{0}$ is a zero-shear rate viscosity represented by a WLF-type model to take into account the temperature and pressure effects : 


$$
\eta_{0}(T, p)=D_{1}\left[-\frac{A_{1}\left(T-T^{*}\right)}{A_{2}+\left(T-T^{*}\right)}\right]
$$

where $A_{1}, A_{2}$, and $D_{1}$ are material constants, and $T^{*}$ is taken as the glass transition temperature which can vary with pressure through $D_{3}$ :

$$
T^{*}=D_{2}+D_{3} p
$$

For tracking the fluid flow front a level set method is employed:

$$
\frac{\partial f}{\partial t}+\nabla \cdot(\boldsymbol{u} f)=0
$$

Where $f$ is the volume fraction, where $f=0$ is the air phase, and $f=1$ is the polymer melt phase. The melt front is tracked by the elements with $0<f<1$. The equations are solved numerically with a finite volume scheme using a 3D unstructured boundary layer mesh (see Fig. 4). For the purpose of this study, the commercially available software Moldex3D Solid R13 was employed.

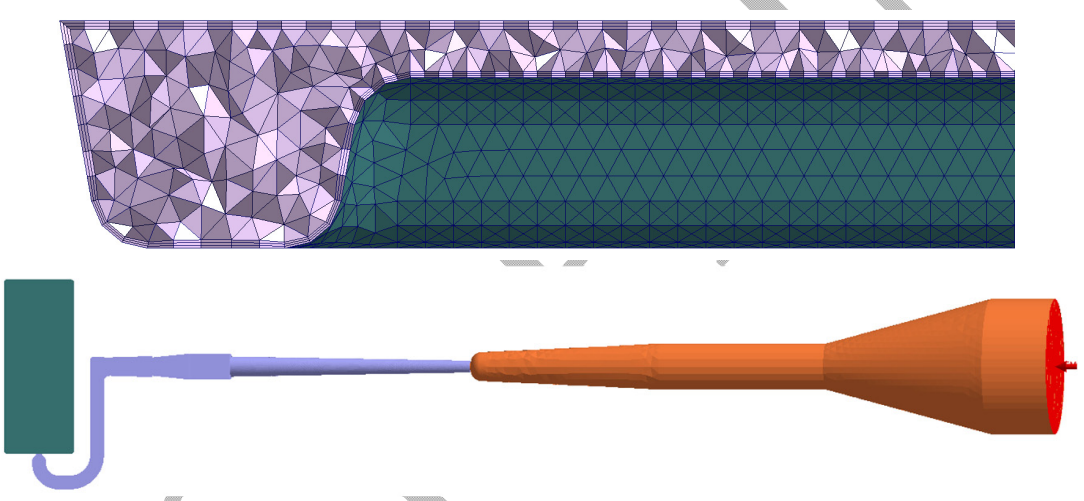

Fig. 4 - Top: Hybrid mesh consisting of tetrahedron elements in the main body and prism elements at the boundary, to better capture larger gradients. The purple elements are inside the cavity, and the green are on the surface. Bottom: Model of the cavity (green), runner (blue), hot runner (orange).

\subsection{Velocity- to pressure-control switch-over}

The high speed movies can give accurate information regarding the melt flow front position with respect to time. However, one challenge is represented by the determination of the point (in terms of both flow front position and time) at which the velocity-control to pressure-control (V/P) switch-over happens, since it is not clearly evident from the high speed camera movies. On the other hand, the determination of the $\mathrm{V} / \mathrm{P}$ is necessary in order to obtain an accurate implementation of the simulation and therefore to be able to compare the simulated flow front pattern with the high speed camera video both during the filling and the packing phases. In order to overcome this issue, the $\mathrm{V} / \mathrm{P}$ switch-over point was found by performing a simulation with high percent filled point (e.g. 98\%), and then noticing the time at which it hits the first and second thermocouples T1 and T2. By looking at the experimental temperature measurements at T1 and T2, and in particular at the points at which the thermal signal rises from both thermocouples, the time needed by the melt to travel between those two points was found. The two timings $t(\mathrm{~T} 1)$ and $t(\mathrm{~T} 2)$ are then 
subtracted and the time interval $\mathrm{t}(\mathrm{T} 1 \rightarrow \mathrm{T} 2)$ between the two thermocouples is determined. The time needed by the melt to travel from the thermocouple $\mathrm{T} 2$ until the V/P switch-over point is then found by looking at the pressure sensor P1 signal. P1 and T2 share the exact same position and their signal is synchronized in time, i.e. $t(P 1)=t(T 2)$. The time interval $t(P 1 \rightarrow V / P)$ between $P 1$ and $V / P$ is then defined as the time between the P1 pressure signal rise (located at same points as T2) until there is a drop in the P1 pressure signal (i.e. the point when the packing phase is initiated). This point is characterized by the time at which the $V / P$, i.e. $t(T 1 \rightarrow T 2)+t(P 1 \rightarrow V / P)$ is happening in the simulation. The pressure at that time is then noted down, and used as the pressure at which the $V / P$ switch-over is taking place.

This was not possible to be obtained in the reference simulations simply due to the fact that the filling time was so fast that the V/P switch-over would not be towards the end of the filling phase, but instead rather later into the packing phase. When the acceleration of the sprue was taken into account the time to fill increased. The overall filling time in the simulation was still too low, but increasing. Adding a hot runner to represent the molten material present before the nozzle added much more time, as this large cushion of material is being compressed, with the effect of slowing down the filling. These aspects of the simulations are discussed in detail in the next paragraph.

\subsection{Setup of initial and improved simulations}

The first simulation conducted showed large time differences compared to the experiments, as the simulation filled too fast. This will be referred to as the reference simulation, and this was implemented with the mentioned ram speed, the temperatures and the different (packing, cooling) times specified in the experimental setup. The size and the geometry of the cushion in front of the sprue and up to the nozzle can have a significant impact on the filling simulation of the cavity and must be taken into account [12][13][14]. This is of particular importance when the volume of the cushion is larger than the runner/cavity system to be molded. This is indeed the case for these experiments conducted in the present study, as the screw diameter is of $35 \mathrm{~mm}$ as previously mentioned. The stroke length of the piston was around $8.5 \mathrm{~mm}$ resulting in an injection volume of approximately $8.2 \mathrm{~cm}^{3}$, which is significantly larger than the volume of cavity and runner which is around $3.2 \mathrm{~cm}^{3}$. This means that all of the molten material standing in front of the screw will be subjected to a certain degree of compression during the injection phase, meaning that the flow rate will slowly increase in the nozzle due to the melt compressibility. This effect results in a higher filling time in experiments than in the reference simulation.

To circumvent this problem, the following different simulation setups were employed to account for the compressibility of the melt volume in the cushion and in the nozzle:

1. Reference simulation, using the ram speed profile from the machine and the geometry of the cavity and runner;

2. Added acceleration of the ram speed;

3. Added nozzle and machine geometry represented as a hot runner to account for the effect of the compressible molten mass located before the runner, see Fig. 4 (bottom);

4. Both added acceleration and nozzle and machine geometry;

5. Same as point 4., but with slightly lower melt temperature in the case of HVPC. 
The simulations were run on an Intel Core i7 $2.5 \mathrm{GHz}, 16 \mathrm{~GB}$ random access memory, with a total number of 1 million elements. Approximately 8 hours were needed to run each simulation.

Simulation results showed that including the screw acceleration gave better overall filling time compared to the actual injection time in the experiments, and better prediction of the pressure curves (see Fig. 8 and Fig. 9).

The use of a hot runner to simulate the cushion of melt is justified, since a hot runner assumes that it is filled with molten polymer, which is actually at the temperature of the melt, as it happens in the barrel of the injection molding machine. The melt might have decreased in temperature before hitting the nozzle, but the assumption is good enough for ABS, due to low sensitivity to temperature of its viscosity. For the HVPC the melt temperature might be slightly lower than expected, and since the viscosity of this polymer exhibits high sensitivity temperature $\left(10-15^{\circ} \mathrm{C}\right.$ lower melt temperature is enough to cause this to be 1.5 times more viscous), a slightly lower melt temperature was included in the simulation for HVPC.

Other effects investigated were transient thermal setting of the mold temperature, pressure dependent viscosity and wall slip. They will be explained in detail in the following.

\subsubsection{Transient thermal simulation}

Using a transient cooling simulation prior to the filling simulation, and then using the calculated temperature distribution in the mold during filling, is possible. In the case of ABS the cooling water was $17^{\circ} \mathrm{C}$ and located $12 \mathrm{~mm}$ from the cavity surface. This does lead to slightly higher temperature at the cavity, of around $22^{\circ} \mathrm{C}$, calculated from the transient cooling phase, and seen in the temperature measurement of thermocouple T3. The improvement was small for ABS, and it was satisfactory to run the simulation assuming such constant temperature for the mold. For HVPC the cooling water was at $95^{\circ} \mathrm{C}$ and the transient simulation proved its temperature to be around $90^{\circ} \mathrm{C}$. This temperature was confirmed by the measurement from the thermocouple T3 during the experiments with HPVC.

\subsubsection{Pressure dependent viscosity}

Pressure dependent viscosity has proved to have an effect on the filling pressure required, and the packing pressure levels. This is shown by Pantani et al. [12] and Guerrier et al. [14] to give a higher pressure drop through the melt, giving more realistic pressure predictions. The D3 coefficient from Eq. 8 was calculated by the method presented by Pantani et al. [12] and simulations were carried out. This did in essence increase the pressure required to fill the cavity due to ram speed prescribed boundary condition.

\subsubsection{Wall slip}

The HVPC material was observed to not properly stick to the mold wall during injection (i.e. the zero velocity at wall condition could probably not be applied throughout the whole simulation). Hence the possibility of wall slip occurrence was considered. This phenomenon could be seen in one of the high speed videos recorded during the experiments with HVPC, in which a certain amount of jetting just after the melt enters the gate was visible (see Fig. 11). A wall slip type boundary condition can be enabled in the software. This option showed that by using a very low friction coefficient of 0.1 , the melt would fill the mold more easily, as lower pressures are required, and the polymer slides in the $0.5 \mathrm{~mm}$ thick section. However, this behavior 
was not observed in the high speed videos. A small improvement in the simulation was obtained by using a higher friction coefficient of 0.7 , but not to the extent as of using a lower melt temperature.

\subsection{Results and discussion}

The flow is characterized by passing through different sections of characteristic geometries. After the first point (i.e. Zero point in Fig. 5), the following points are then determined to characterize the different filling steps:

- Point $\mathrm{A}$ is positioned in correspondence to the first corner in the runner, and the time is defined as the moment at which the melt completely fills up the corner.

- Point B is defined when the melt is at the gate, i.e. when it is just about to enter the cavity.

- Point $\mathrm{C}$ is indicated as when the melt has entered the cavity and the two first corners are filled.

- Point D indicates when the two melt fronts start to form a weld line, meeting after the $0.5 \mathrm{~mm}$ thick section.

- Point $\mathrm{E}$ is defined as when the melt has flowed over the location of the last thermal sensors T3.

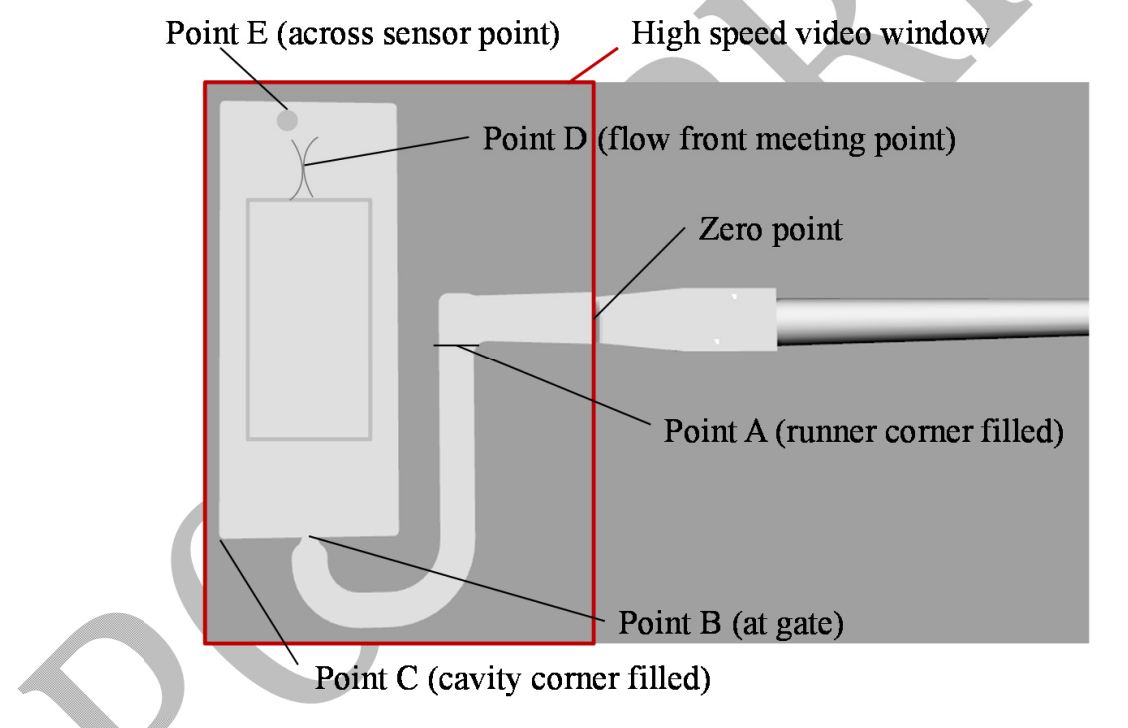

Fig. 5 - Points that indicate the timing measurements.

These timings are plotted in Fig. 6 and Fig. 7 as the interval between the points and the total time from the zero point. The total time reflects the total time the melt is visible in the high speed video field of view, i.e. it is shorter than the total experimental injection time of $1 \mathrm{~s}$. As seen in Fig. 6 for the ABS, the reference simulation tends to fill faster than in the experiment. Adding the acceleration of the screw does seem to improve the results close to the timings of the high speed video, and it does increase the total injection time close to the real time. An actual improvement is seen when the hot runner geometry for the nozzle and barrel is included. Now the timings are within 10-15 ms for the intervals, and 20-25 ms for the total video 
time. The result is that by using both acceleration and nozzle/barrel hot runner it is possible to achieve a good agreement for the high speed video timings and the total injection time with the experiments.

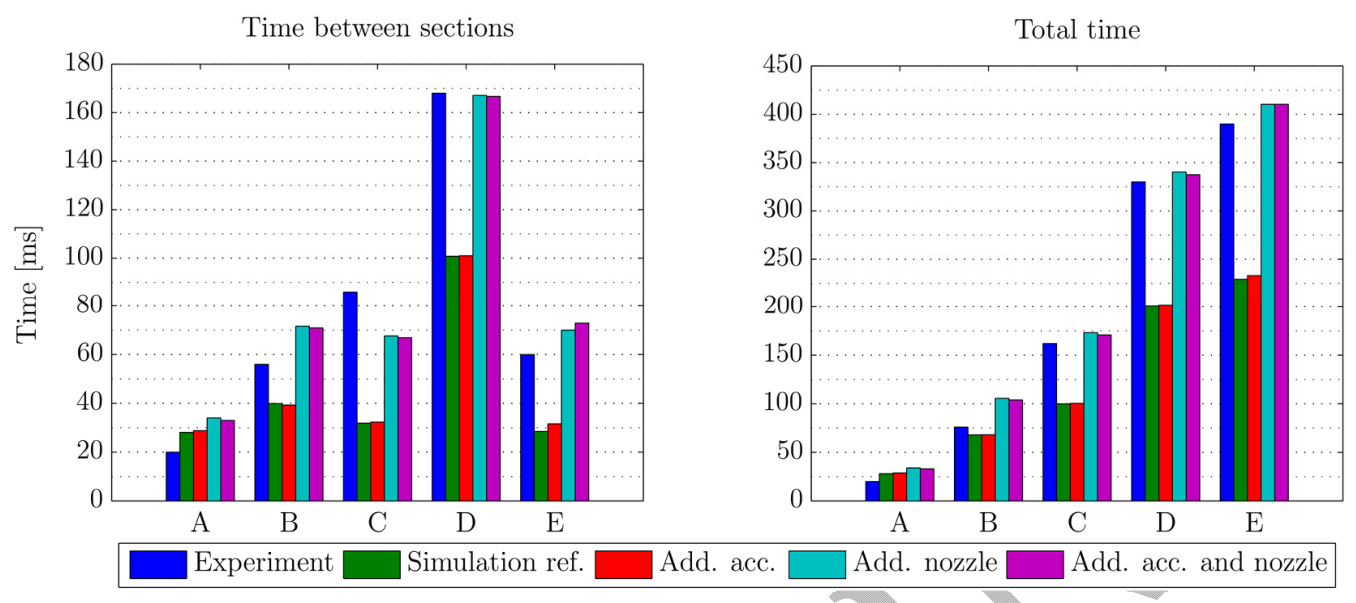

Fig. 6 - Experimental and simulated timings compared for ABS

In Fig. 7 the same results are represented for HVPC. The same trend is seen as with ABS, but there is still a difference in both timings when only using the acceleration and the nozzle/barrel hot runner. This is improved when using a lower melt temperature, as the polymer seems to solidify and block the flow before the packing phase starts.

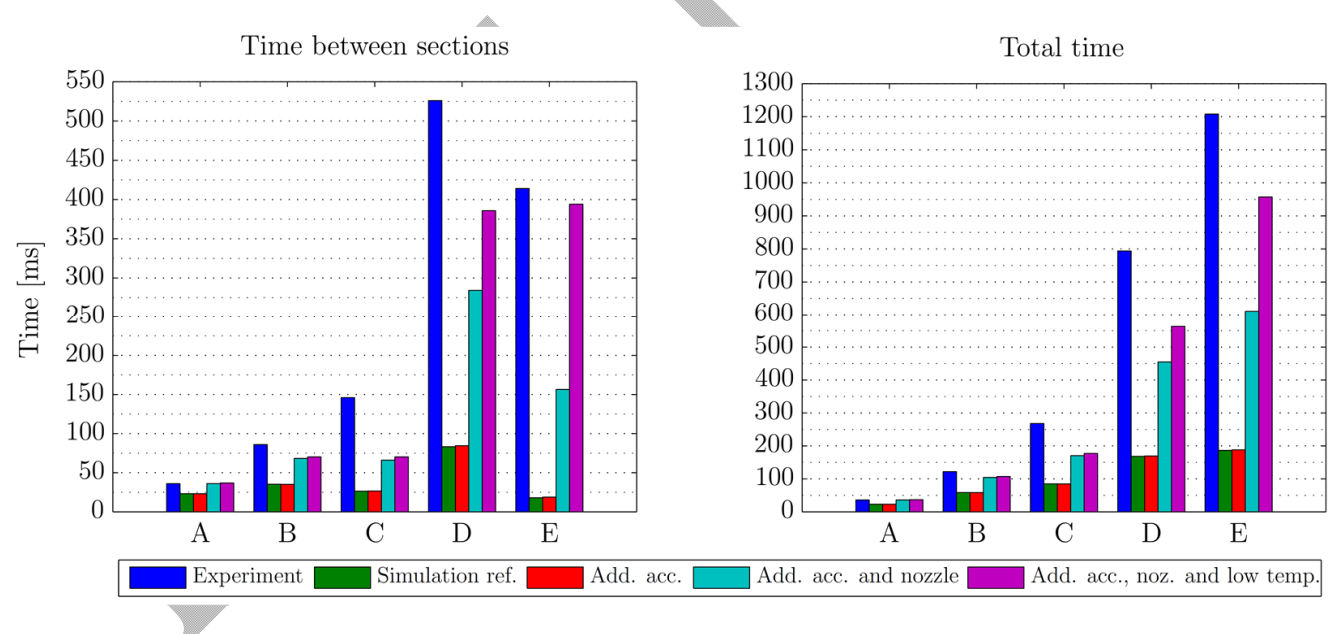

Fig. 7 - Experimental and simulated timings compared for HVPC

The pressure curves from the simulation at the sensor location can be seen in Fig. 8 for ABS and in Fig. 9 for HVPC. As shown, the pressure curves are much steeper in the reference runs. When the acceleration is added it gives a flat pressure increase in the beginning, also giving a longer filling time. Adding the cushion compression, the pressure increase is smoother, as seen in the experiments. Finally, a very good agreement is obtained for ABS using both acceleration and the cushion, as the pressure curve in Fig. 8 corresponds to the experimental curve shown in Fig. 3. For the HVPC, using a slightly lower melt temperature seemed to 
improve the results further, but there is still a visible deviation from the experimental results. Also, as seen in the figure of the high speed video in Table 2, there is a solidified tip of the material coming into the runner first, and getting stuck there. This cold plug might make it more difficult for the material to flow in the experiments than in the simulations. Furthermore, it seems that the HVPC is more prone to wall slipping, which was very apparent in the experiments performed at higher injection speed, see Fig. 11. As can be seen in the images, the HVPC showed the tendency to produce jetting after the gate.

For a qualitative comparison of the simulations and experiments, the high speed videos and simulations shown in Table 1 and Table 2 for the points A-E and the 'END OF FILL' point (defined as when the melt fills the two end corners, as the V is not completely filled for HVPC) are considered. The timings are noted at the same points at those in Fig. 6 and Fig. 7. The visual comparison seems to be in good agreement, especially for ABS, as the V-shape of the weld line is almost identical. In the case of the HVPC, the V-shape of the weld line seems to be smaller, indicating that if has slightly more flowability with respect to the experiments. These observations are also confirmed when comparing the experimental timings with those obtained in the simulations (see Table 3).

Reference

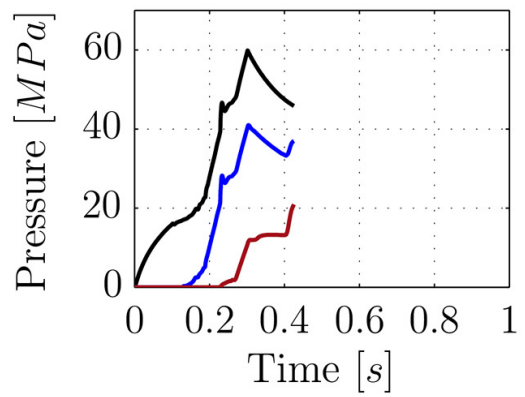

Nozzle/barrel geo.

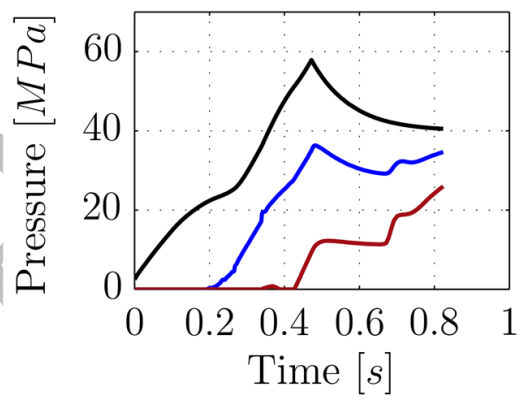

Acceleration

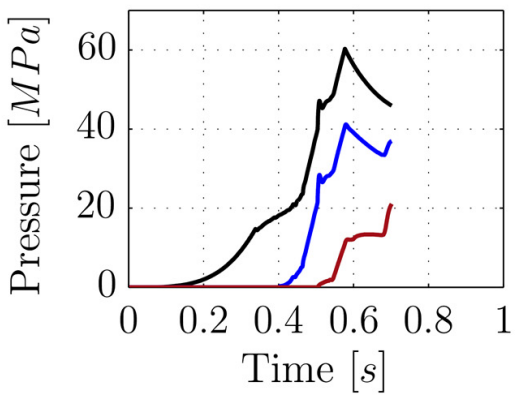

Acc. and noz./bar. geo.

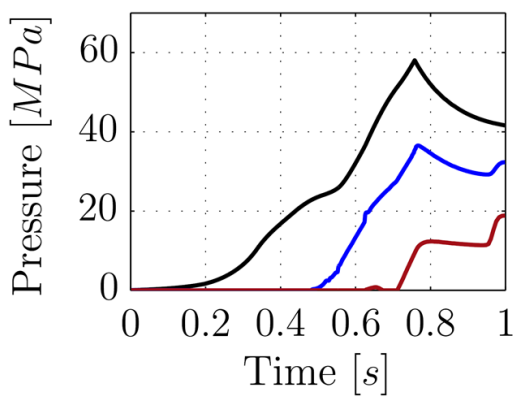

$$
-\mathrm{P} 0-\mathrm{P} 1-\mathrm{P} 2
$$

Fig. 8 - Simulated pressure curve for ABS at the two pressure sensor location, and the boundary pressure on the runner/hot runner denoted P0. 

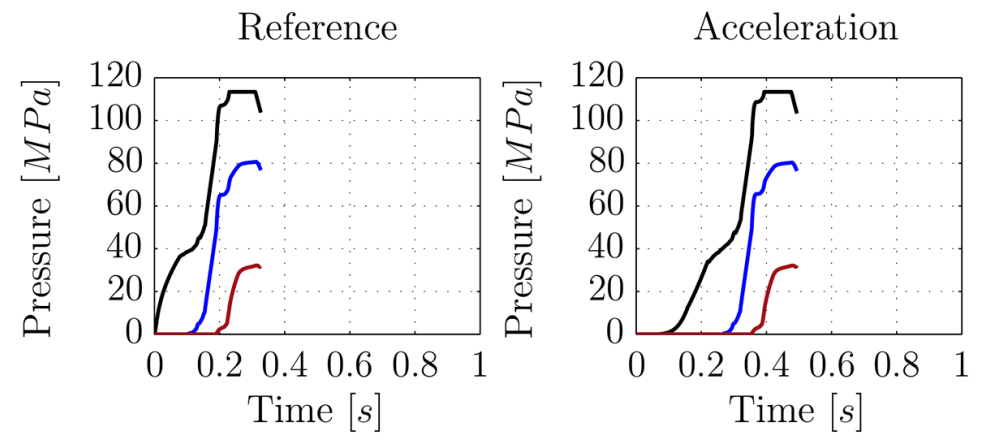

Acc. and noz./bar. geo. Acc., noz. and low melt temp.
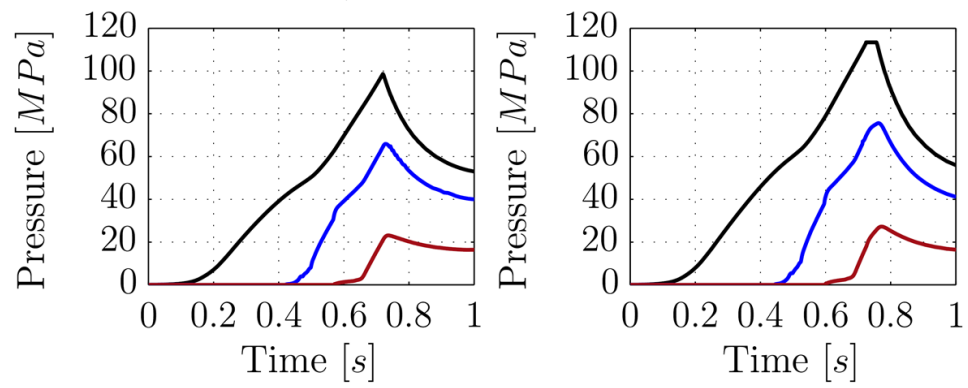

$$
-\mathrm{P} 0-\mathrm{P} 1-\mathrm{P} 2
$$

Fig. 9 - Simulated pressure curve for HVPC at the two pressure sensor location, and the boundary pressure on the runner/hot runner denoted P0.
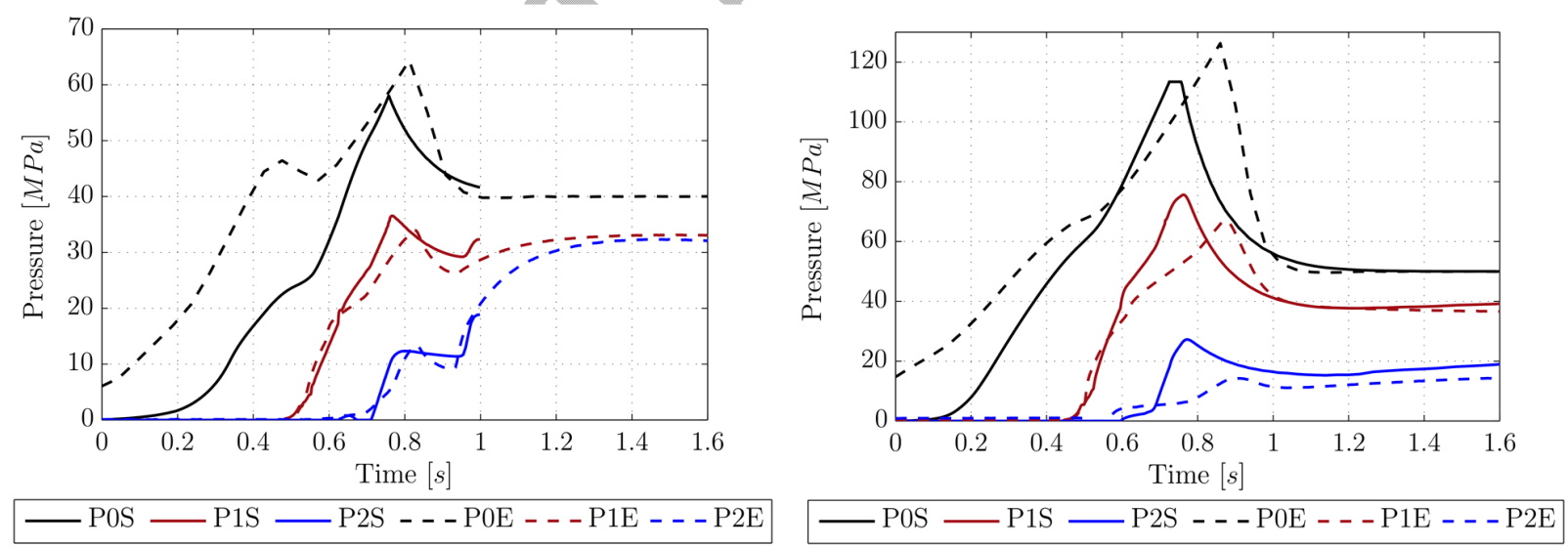

Fig. 10 - Experimental and simulated pressures P0, P1, P2 for ABS (left) and HVPC (right) obtained with the best simulation setting. ' $S$ ' denotes simulation, and ' $E$ ' experiments. 
Table 1 - Experiments and simulation results of flow front position, pattern shape and filling time with ABS. The time zero point is set when the flow enters the field of view of the high speed camera. The simulation includes both acceleration and hot runner nozzle geometry and melt cushion.

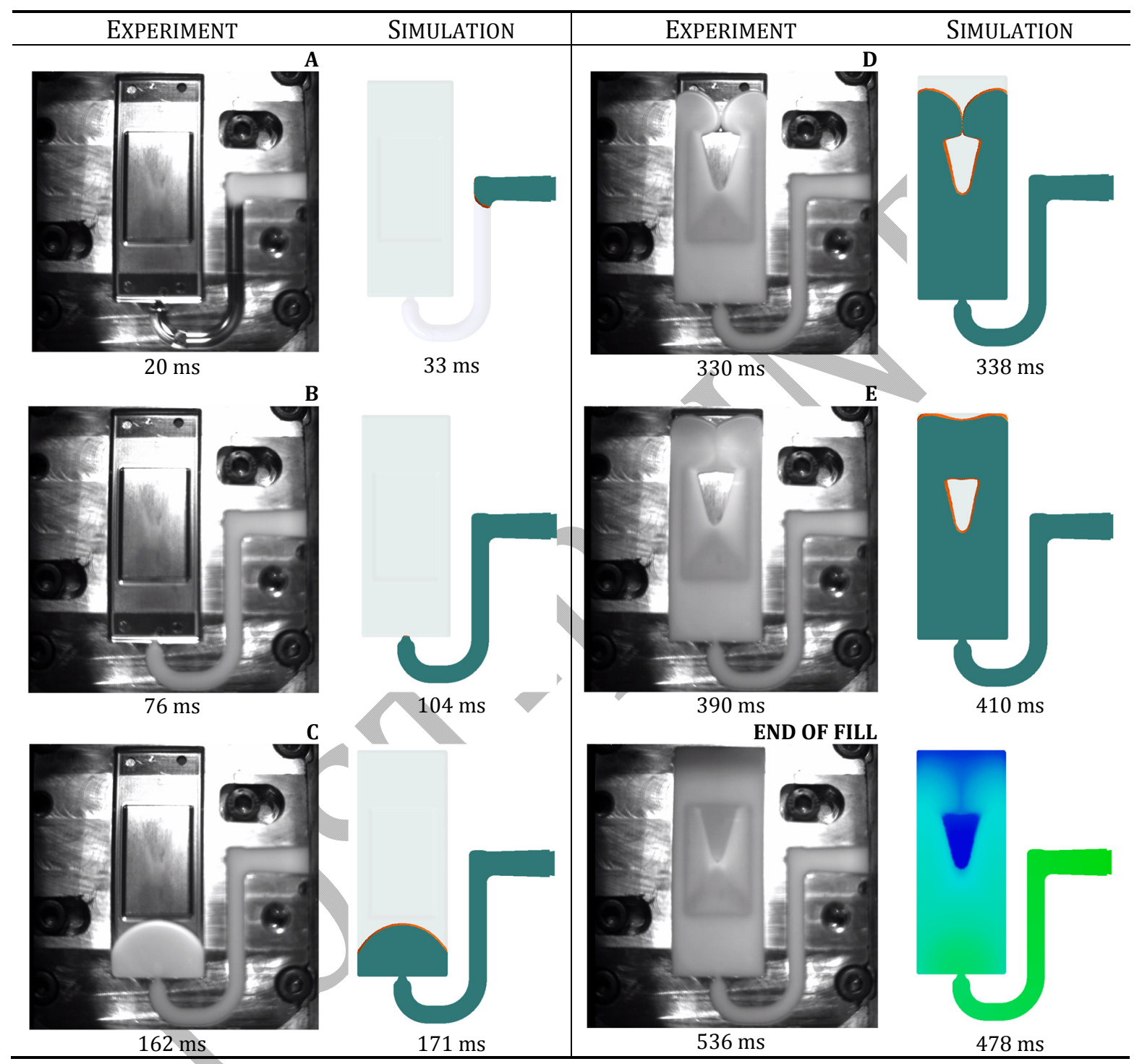


Table 2 - Experiments and simulation results of flow front position, pattern shape and filling time with HVPC. The time zero point is set when the flow enters the field of view of the high speed camera. The simulation includes both acceleration and hot runner nozzle geometry and melt cushion.

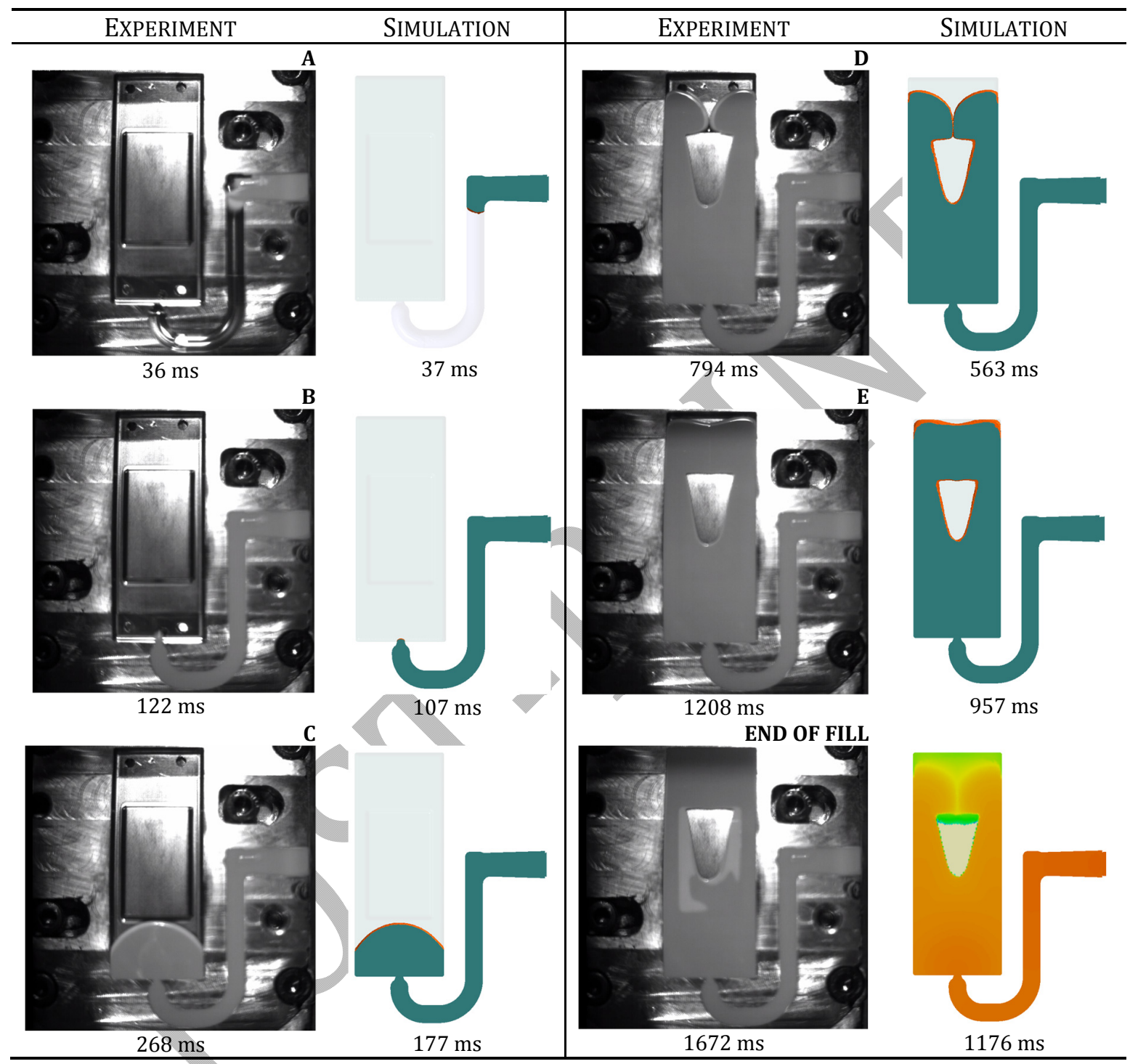


Table 3 - Absolute and relative deviations of simulated times with respect to experiments. Absolute deviation $\Delta \mathrm{t}=\mathrm{t}($ simulation $)-\mathrm{t}($ experiments $)$; relative deviation $\Delta \%=\Delta \mathrm{t} / \mathrm{t}($ experiments $) \cdot 100$.

\begin{tabular}{ccc|cc}
\hline \multirow{2}{*}{ SECTION } & \multicolumn{2}{c|}{ ABS } & \multicolumn{2}{c}{ HVPC } \\
\cline { 2 - 5 } & ABSOLUTE DEVIATION & RELATIVE DEVIATION & ABSOLUTE DEVIATION & RELATIVE DEVIATION \\
\hline A & $13 \mathrm{~ms}$ & $65,0 \%$ & $1 \mathrm{~ms}$ & $2,8 \%$ \\
B & $28 \mathrm{~ms}$ & $36,8 \%$ & $-15 \mathrm{~ms}$ & $-12,3 \%$ \\
$\mathbf{C}$ & $9 \mathrm{~ms}$ & $5,6 \%$ & $-91 \mathrm{~ms}$ & $-34,0 \%$ \\
$\mathbf{D}$ & $8 \mathrm{~ms}$ & $2,4 \%$ & $-231 \mathrm{~ms}$ & $-29,1 \%$ \\
E & $20 \mathrm{~ms}$ & $5,1 \%$ & $-251 \mathrm{~ms}$ & $-20,8 \%$ \\
END OF FILL & $-58 \mathrm{~ms}$ & $-10,8 \%$ & $-496 \mathrm{~ms}$ & $-29,7 \%$ \\
\hline
\end{tabular}
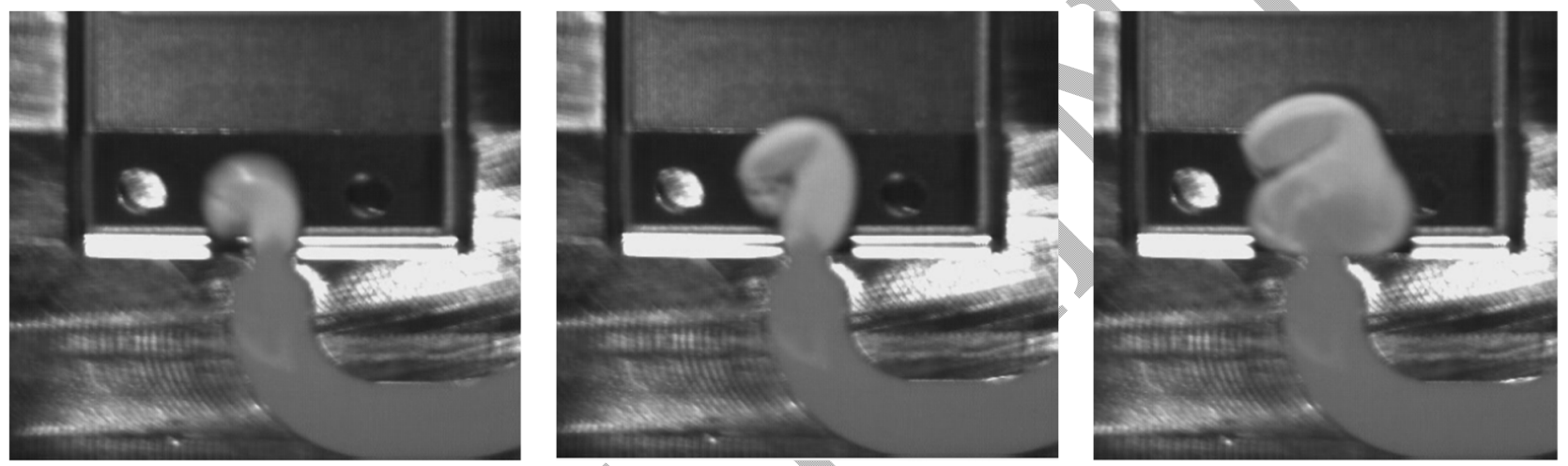

Fig. 11 - Jetting occurring just after the gate at $42 \mathrm{~ms}, 46 \mathrm{~ms}, 54 \mathrm{~ms}$, after the time zero point.

\section{Conclusion}

The use of a glass mold is an effective method of performing a direct visual comparison of injection molded parts with simulations. This was achieved in the present study in terms of visual filling pattern and also in terms of actual timing of the melt front during filling and packing. The mold design with a perpendicular injection plane to the mold opening direction presented in this work provided a convenient camera positioning. Thermocouples and pressure sensors were installed and helped getting the overall filling times correct, that are not captured by the high speed camera, and obtaining valuable information regarding the velocity- to pressure-control switch-over, since this is not visible in the video recordings. Simulations have been conducted using the machine inputs and compared with timings from the high speed video and pressure sensors. The reference (i.e. default) simulation showed large deviations of the timings compared with the experimental high speed videos. The reason was mainly due to the cushion size for the moldings which is larger than the volume of the part and runner volume. The other effect was the inclusion of the barrel acceleration in the machine input data, which increased the injection time. Without using both effects the simulation was filling the runner and cavity too easily, resulting in a lower filling time than that seen in the experiments. The most accurate simulation results showed simulations deviations within 10-30 ms (relative deviation in order of $5-10 \%$ ) for the ABS and slightly more for the high viscosity PC, in the range 
of 100-500 ms (relative deviation in order of 20-30\%) on timings between different sections during filling. The reason for the HVPC not being as close to the experiments as ABS, is suspected be that the viscosity is more sensitive to temperature, it showed some wall slip and that a cold plug is observed in the runner in the high speed videos. The pressure levels from the sensors agree very well for ABS, and for HVPC there is a slight deviation, mostly in the timings, since the curvature and the levels of the pressure profiles are in fairly good agreement with the experiments.

\section{Acknowledgment}

This work was carried out as part of the Innomold Project (Innovative Plastic Products and More Energy Efficient Injection Molding Processes) supported by the Danish Council for Technology and Innovation.

\section{References}

[1] S. Yang, S. Nian and I. Sun, "Flow Visualization of Filling Process during Micro-Injection Molding," Intern. Polymer Processing, vol. 4, pp. 354-360, 2002.

[2] S. C. Nian and S. Y. Yang, "Flow Visualization of Filling with Aid of Colored Billets During Impact Micro-Injection Molding," Intern. Polymer Processing, vol.4, pp. 402-407, 2004.

[3] X. Han and H. Yokoi, "Visualization Analysis of the Filling Behavior of Melt into Microscale V-Grooves During the Filling Stage of Injection Molding," Polym. Eng. Sci., vol. 46, pp. 1590-1597, 2006.

[4] S. Hasegawa and H. Yokoi, "Visualization of Melt-Flow Behavior Inside the Runner in Ultra High Speed Injection Molding," Intern. Polymer Processing, vol. 5, pp. 464-472, 2006.

[5] G. S. Layser and J. P. Coulter, "Effect of Processing Parameters on Flow Hesitation with Glass-Filled Reinforced Thermoplastics: Experimental Investigation with Direct Visualization," ANTEC, pp. 342$348,2008$.

[6] R. Spares, B. R. Whiteside and P. D. Coates, "High Speed Thermal Imaging of Micromoulding," ANTEC, pp. 2570-2573, 2009.

[7] B. Whiteside, R. Spares and P. Coates, "Rheo-Optical Measurements in Micromoulding," ANTEC, pp. 1803-1810, 2009.

[8] H. Yokoi and D. Yoshida, "Ultra-High-Magnification Visualization of Melt Filling Behaviors in Line \& Space Replication Molding Using a Microscope Built-in Mold," PPS, vol. 27, 2011.

[9] A. S. Yanev, G. R. Dias and A. M. Cunha, "Direct Visualization of Conventional Injection Molding," ANTEC, pp. 1097-1101, 2007. 
[10] A. S. Yanev, G. R. Dias and A. M. Cunha, "Visualization of Injection Moulding Process," Materials Science Forum, Vols. 587-588, pp. 716-720, 2008.

[11] P. Dvorak, T. Barriere and J. C. Gelin, "Direct observation of mould cavity filling in ceramic injection moulding," Journal of the European Ceramic Society, vol. 28, pp. 1923-1929, 2008.

[12] U. Vietri, A. Sorrentino, V. Speranza and R. Pantani, "Improving the Predictions of Injection Molding Simulation Software," Polym. Eng. Sci., vol. 51, pp. 2542-2551, 2011.

[13] G. Tosello, F. Costa and H. Hansen, "Micro Injection Moulding High Accuracy Three-Dimensional Simulations and Process Control," Proceedings of Polymer Process Engineering 11 - Enhanced Polymer Processing, pp. 413-435, 6-7 December 2011.

[14] P. Guerrier, G. Tosello and J. H. Hattel, "Analysis of Cavity Pressure and Warpage of Polyoxymethylene Thin Walled Injection Molded Parts: Experiments and Simulations," Proceedings of 30th International Conference of the Polymer Processing Society (PPS-30), Cleveland, OH, p. 5 pages, 8-12 June 2014. 